\title{
ON THE CYCLIC J-INTEGRAL OF A 3D SEMI ELLIPTICAL SURFACE CRACK
}

\author{
Ross Beesley \\ Department of Mechanical \& Aerospace Engineering, \\ University of Strathclyde, \\ Glasgow, UK \\ Haofeng Chen \\ Department of Mechanical \& Aerospace Engineering, \\ University of Strathclyde, \\ Glasgow, UK
}

\author{
Martin Hughes \\ Siemens Industrial Turbomachinery, \\ Lincoln, UK
}

\begin{abstract}
This paper investigates an approach for calculating the cyclic J-Integral $(\Delta \mathrm{J})$ through a new industrial application. A previously proposed method is investigated further with the extension of this technique through a new application of a practical 3D notched component containing a semi-elliptical surface crack.

Current methods of calculating the cyclic J-Integral are identified and their limitations discussed. A modified monotonic loading method is adapted to calculate the cyclic Jintegral of this 3D Semi Elliptical Surface Crack under cyclic loading conditions. Both the finite element method (FEM) and the Extended Finite Element Method (XFEM) are discussed as possible methods of calculating the cyclic J-Integral in this investigation. Different loading conditions including uni-axial tension and out of plane shear are applied, and the relationships between the applied loads and the cyclic J-integral are established. In addition, the variations of the cyclic J-integral along the crack front are investigated. This allows the critical load that can be applied before crack propagation occurs to be determined as well as the identification of the critical crack direction once propagation does occur.

These calculations display the applicability of the method to practical examples and illustrate an accurate method of estimating the cyclic J-integral.
\end{abstract}

Keywords: crack, J-integral, cyclic J-integral, fracture mechanics, FEA, XFEM

\section{NOMENCLATURE}

$\begin{array}{ll}\text { A } & \text { Dowling and Begley Fatigue Law Constant } \\ \mathrm{C} & \text { Paris' Law Constant } \\ \mathrm{da} & \text { Change in Crack Length } \\ \mathrm{dN} & \text { Change in Number of cycles } \\ \mathrm{J} & \text { J-Integral } \\ \Delta \mathrm{J} & \text { Cyclic J-Integral }\end{array}$

$\begin{array}{ll}\text { Jmax } & \text { J-Integral at maximum cyclic load } \\ \text { Jmin } & \text { J-Integral at minimum cyclic load } \\ \mathrm{K} & \text { Stress Intensity Factor } \\ \Delta \mathrm{K} & \text { Stress Intensity Factor Range } \\ \mathrm{Kmax} & \text { Stress Intensity Factor at maximum cyclic load } \\ \mathrm{Kmin} & \text { Stress Intensity Factor at minimum cyclic load } \\ \mathrm{m} & \text { Paris' Law Constant } \\ \mathrm{MPa} & \text { Mega Pascals } \\ \mathrm{N} & \text { Newtons } \\ \varepsilon & \text { Strain } \\ \sigma & \text { Stress }\end{array}$

ABBREVIATIONS

$\begin{array}{ll}\text { EPFM } & \text { Elastic Plastic Fracture Mechanics } \\ \text { FE } & \text { Finite Element } \\ \text { FEM } & \text { Finite Element Method } \\ \text { LEFM } & \text { Linear Elastic Fracture Mechanics } \\ \text { MML } & \text { Modified Monotonic Loading } \\ \text { RSM } & \text { Reference Stress Method } \\ \text { SIF } & \text { Stress Intensity Factor } \\ \text { XFEM } & \text { Extended Finite Element Method }\end{array}$

\section{$1 \quad$ INTRODUCTION}

Fracture mechanics regards the initiation and propagation of cracks. The impact of material fracture varies depending on the specific application but the results can be catastrophic. Therefore gaining an understanding of fracture and failure is very important. The ability to predict when a crack will initiate and fail, and thus the resulting fatigue life of the component must be understood to ensure the safe design and utilisation of structural components. Fracture mechanics provides generalised techniques that are widely applied to a number of different industries and uses. For this reason, this field of study has attracted a large number of researchers [1][2][3].

Stress raisers are of particular importance when considering engineering components. Design features such as notches or sharp corners, and even minor defects such as scratches and 
corrosion can introduce stress raisers which reduce the critical stress at which crack initiation can occur. Such features can limit the fatigue life since failure can occur at a reduce load or fewer loading cycles. The stress intensity factor (SIF), is a measure of the stress conditions near a crack tip and can be used to predict stress and fracture behaviour under different loading conditions. Extensive experimental testing has permitted the development of a set of standardised equations for calculating the stress intensity factor for a number of different crack and model geometries.

Crack simulation is vitally important and there are a number of methods of using fracture mechanics in order to evaluate fracture and fatigue life including R5 and R6 codes [4][5] and stress intensity factor analysis. However, the focus of this paper is the J-Integral, an alternative to SIF when considering elastic plastic fracture mechanics, and how this can be extended to allow the evaluation of fatigue life. Although a more complex parameter than the stress intensity factor, the development of commercial and bespoke finite element software packages has made the J-Integral a more promising technique and as a result interest in the field has increased in recent years. The importance of this as a fracture mechanics and crack simulation parameter is discussed in this paper. Following discussion of the relevant methods of fracture mechanics, the J-Integral is introduced and methods of the extension of this parameter to allow for cyclic fatigue are investigated through the application of techniques to an industrial test specimen.

\subsection{Objectives}

The overarching aim of this investigation is to assess the suitability of an extended monotonic analysis for approximating the cyclic J-integral. Initially, the limitations with the current methods of determining cyclic J-Integral will be identified. The suitability of the proposed Modified Monotonic Loading (MML) Method will then be assessed. Finally, this technique will be applied to an industrial test specimen in order to calculate the cyclic J-Integral and its variation with increasing load and crack location. This paper is organised as follows:

Section 2 discusses the background theory on numerical methods that exist for addressing crack modelling. In Section 3, finite element methods are introduced and the proposed method for calculating the $\Delta \mathrm{J}$ will be discussed. Section 4 presents the model specific to this application and the associated material and loading properties defined. The investigation continues with Section 5 which presents the obtained results for the validation of the MML technique as well as the calculated cyclic J-Integral variation with increasing load and crack location.

\section{THEORETICAL BACKGROUND}

\subsection{Contour Integrals}

Contour Integration allows the evaluation of integrals along certain paths. Within finite element, contour integration allows the calculation of fracture mechanics parameters of a material including the stress intensity factor, $\mathrm{K}$, and the J-Integral, J. The finite element software package, ABAQUS[6] allows the calculation of such integrals along paths encircling a crack front. While these contour integrals do not themselves directly predict how a crack will propagate, they can be used to provide valuable information and offer some indication as to how the crack may behave as well as the estimation of fatigue life.

\subsection{Fatigue Life}

In order to calculate the fatigue life under Linear Elastic Fracture Mechanics (LEFM), the stress intensity factor, $\mathrm{K}$, is required. The SIF is a function of stress and crack length. Under cyclic loading conditions, the SIF range between maximum and minimum loading can be used to predict fatigue life through Paris' Law[7] as shown in Eq 1.

$\frac{d a}{d N}=C \Delta K^{m}$

Where $a$ is crack length, $N$ is number of cycles, $\Delta K=K_{\max }-K_{\min }$ is the stress intensity factor range and $C$ and $m$ are constants. This relationship holds for LEFM properties, however it does not apply to Elastic-Plastic Fracture Mechanics (EPFM) properties since the relationship becomes less accurate as plasticity levels increase. Therefore, a new parameter is required to allow a similar approach for calculating the fatigue life of elastic-plastic materials. A suitable alternative is the JIntegral, which represents a method of calculating the strain energy release rate per unit area of a fracture surface. The JIntegral offers an EPFM equivalent to the SIF for LEFM. Calculating the J-Integral under monotonic loading conditions is relatively simple. It is done routinely and has proven itself to be a good method of modelling crack behaviour. However, difficulties arise when implementing cyclic loading conditions.

The cyclic J-Integral, $\Delta \mathrm{J}$ is a function of the stress and strain range, $\Delta \sigma$ and $\Delta \varepsilon$ and as a result, unlike the cyclic stress intensity factor, is not simply equal to $\mathrm{J}_{\max }-\mathrm{J}_{\min }$ and therefore $\Delta J \neq J_{\max }-J_{\min }$. For this reason, calculating the cyclic $\mathrm{J}$ Integral is inherently more difficult than for monotonic loading and no standard techniques have yet been developed to determine the cyclic J-integral. In order to determine the EPFM fatigue life, the J-Integral must be extended to allow for cyclic loading conditions, much like the SIF range is used in LEFM cyclic fatigue.

The J-Integral is a measure of the elastic and plastic work done for crack growth to occur and as a result is assumed to be equal to the sum of the elastic and plastic components, as introduced by Sumpter and Turner[8]. At low levels of plasticity, J is dominated by the elastic component and so the linear elastic based strain energy release rate is sufficient for calculating $\mathrm{J}$. However, when the effect of the plastic zone becomes more substantial, this linear elastic approximation is no longer valid. Therefore, since the J-Integral can be used to determine the fatigue life, a reliable method of its calculation is vitally important. 
The cyclic J-Integral was first proposed and implemented by Dowling and Begley[9]. A power law behaviour, similar to that of the Paris equation was developed and so the fatigue crack growth rate of cyclic loading EPFM can be written as:

$\frac{d a}{d N}=A(\Delta J)^{m}$

Where $A$ and $m$ are constants.

\subsection{Limitations of Existing Technologies}

The GE/EPRI[10] and Reference Stress Method (RSM)[11] offer simplified methods of approximating the cyclic J-Integral. However, due to the nature of these methods they exhibit considerable limitations and thus produce overly conservative results. The approximations that are made render these methods unable to assess detail along a 3D crack front. For example, the Reference Stress concept is based on the limit load analysis, and as such, the J-Integral variation is calculated with little consideration of the geometry of the specimen. This provides a greatly approximated value of the induced stress, thus reducing the accuracy of the calculation of the J-Integral. In addition, this method cannot provide three dimensional detail of a crack and so monitoring the crack front variation of the stress intensity factor and J-Integral is not possible. This introduces a major issue with this technique since any variation in crack parameters could be overlooked and hence fail to provide valuable information regarding crack initiation and thus, fatigue life. For this reason, these methods are not considered appropriate for complex 3D industrial applications.

ABAQUS and other FE packages are capable of calculating the J-Integral under monotonic loading, however, they are currently unable to automatically determine the cyclic JIntegral from stress and strain history. Manually calculating the cyclic J-Integral, would require extensive and very time consuming post processing of the analysis history data. Manually calculating in this way is therefore not feasible and so a more automated method is required if it is to be viable technique.

\subsection{Crack Modelling}

Simulating a 3D surface crack is much more complicated than a $2 \mathrm{D}$ crack. The variation of $\Delta \mathrm{K}$ and $\Delta \mathrm{J}$ along the crack front is dependent on the type and magnitude of the applied loading and as a result will vary depending upon the location along the 3D surface crack front. Different locations will result in different values of $\Delta \mathrm{K}$ and $\Delta \mathrm{J}$ and will thus affect the crack propagation direction. It is therefore vitally important to simulate the $\Delta \mathrm{J}$ with a high level of detail under different loading conditions in order to gain an understanding of the crack behaviour and thus predict its propagation.

\section{NUMERICAL METHOD}

Modelling simplified models such as infinite plates and blocks can provide valuable insight into crack behaviour. However, these large simplifications can overlook the complexities of real life applications that are found in industry. Therefore, increasing the model complexity makes computational models more akin to industrial applications and thus the results can offer more value than that of greatly simplified cases. This provides reason for modelling a complex geometry test specimen in this investigation.

\subsection{Finite Element Analysis (FEA) Crack Simulation}

FEA allows the modelling of cracks and the calculation of their associated parameters such as SIF and J-Integral. Within ABAQUS, cracks can either be modelled using the traditional Finite Element Method (FEM) or the Extended Finite Element Method (XFEM) [12].

FEM crack modelling requires the definition of contour integral crack properties which allow the crack front and the crack extension direction to be specified. In addition, a seam crack must be implemented which allows the fracture surfaces to separate. This method currently only offers modelling of stationary cracks, meaning that propagation cannot automatically be modelled unlike XFEM. Within FEM, mesh quality around the crack tip is critical and must be refined sufficiently to allow for acceptable accuracy which increases the computational modelling and analysis effort.

\subsection{Extended Finite Element Method (XFEM)}

The extended finite element method is capable of modelling mesh independent cracking, meaning that crack initiation and propagation can be modeled without prior definition. A propagating crack does not need to adhere to element boundaries unlike the traditional finite element method (FEM). This reduces the importance of mesh refinement in the region of the crack front. XFEM can model stationary or propagating cracks, however ABAQUS is currently only capable of determining crack parameters such as SIF and J-Integral for stationary cracks. This method is still in its infancy but it shows a great deal of potential. It provides a simple method of modeling complex crack geometries without the need for extensive mesh refinement which can be very computationally expensive both in implementation and analysis. This method is capable of calculating contour integrals such as the SIF and JIntegral, however, when a high level of geometrical detail is introduced, the accuracy of contour integration close to the crack tip is compromised. For this reason, XFEM will not be used as a technique for calculating contour integrals in this investigation and traditional FEM will be used instead.

\subsection{Modified Monotonic Loading (MML) Method}

A method has been proposed which provides a reasonable approximation for the calculation of the cyclic J-Integral which addresses the known issues in the existing technologies. This can be achieved through modification of a monotonic loading analysis by replacing $\sigma_{y}$ with $2 \sigma_{y}$ and replacing the cyclic load 
range with a single monotonic load equal to the range. This allows such a modified monotonic analysis to replicate the conditions of a cyclic loading analysis. This method is referred to in this paper as the Modified Monotonic Loading (MML) Method. This follows on from the work of Chen and Chen[13]. It was discovered that in an un-cracked body subjected to variable loading conditions, the differences between this MML method and the equivalent cyclic analysis were relatively small. Their work indicated the potential for this technique as a method of determining the cyclic J-Integral. In this investigation, this MML method will be investigated and tested further on an industrial test specimen.

Since the cyclic J-Integral is a function of stress and strain range (4), for this method to be viable and the hypothesis that it is capable of accurately replicating a cyclic loading analysis to hold true, then the stress range and strain range data from a cyclic loading analysis must match the stress and strain data from the modified monotonic loading analysis (5) (6).

Using this assumption will then allow the determination of the cyclic J-Integral through the MML method within ABAQUS. Following such a hypothesis, the cyclic J-Integral values under fatigue loading can be assumed to be equal to the J-Integral values from the Modified Monotonic Loading method analysis (7). It is assumed that:

$J=f(\sigma, \varepsilon)$

$\Delta J=f(\Delta \sigma, \Delta \varepsilon)$

Therefore, if

$\sigma_{M M L}=\Delta \sigma_{\text {cyclic }}$

And

$\varepsilon_{M M L}=\Delta \varepsilon_{\text {cyclic }}$

Then,

$J_{M M L}=\Delta J_{\text {cyclic }}$

Therefore, for a cyclic loading analysis of load range $\pm \mathrm{X} \mathrm{MPa}$, the cyclic J-Integral can be approximated by performing a Modified Monotonic Loading analysis with a single load of $2 \mathrm{X}$ $\mathrm{MPa}$. It is important to note that the MML method does not apply to a non-zero amplitude where the load range does not equal $\pm X$, for instance between 0 and $+X$.

\section{NUMERICAL APPLICATION}

\subsection{Finite Element Model}

The finite element software package, ABAQUS was used for the computational analyses performed in this investigation. Within ABAQUS, a notched industrial test specimen as presented by Leidermark[14] was modelled with appropriate model partitioning employed. This allowed the implementation of a refined mesh around the most critical region of the notch, whilst a more coarse mesh was modelled in the less critical regions. A 3D semi-elliptical surface crack with a semi-major axis radius of $1 \mathrm{~mm}$ and a semi-minor axis radius of $0.75 \mathrm{~mm}$ was modelled with a focused mesh swept along the crack front to allow for improved accuracy. Cyclic and Modified Monotonic Loading analyses were performed and the results of each compared in order to assess the suitability of the method. Once validated, additional MML analyses were performed to calculate the cyclic J-Integral under uni-axial and out of plane shear loading.

The finite element mesh of the test specimen is shown in Fig 1 and a close-up of the opened crack surfaces shown in Fig 2.

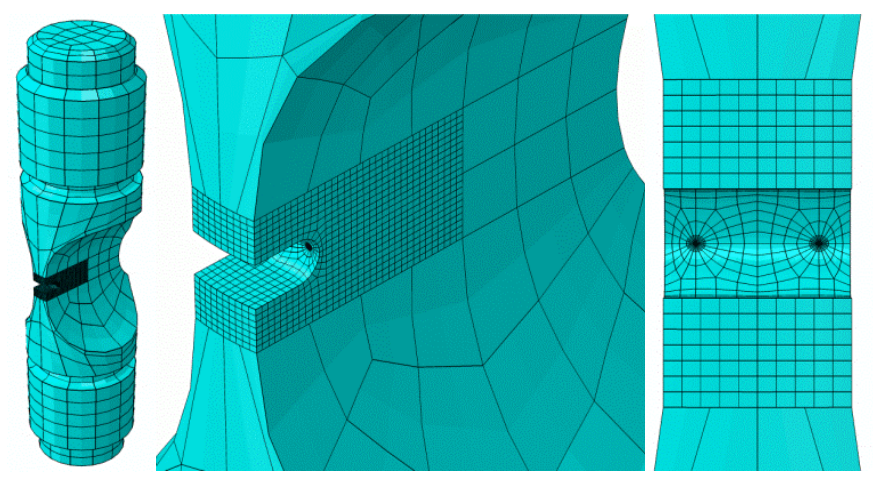

Fig 1: Finite element mesh of structure showing entire specimen (a) and close up view of notch (b) and (c)

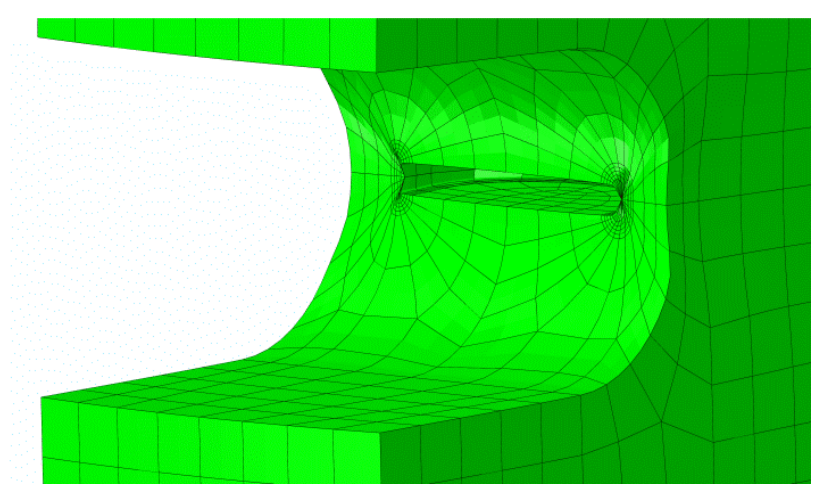

Fig 2: Open crack surfaces

\subsection{Material Properties \& Loading Conditions}

A nickel based super alloy similar to those in turbine applications was used in this investigation with a Young's Modulus of 207GPa, Poissons' Ratio of 0.29 and Yield Stress of $1000 \mathrm{MPa}$.

The accuracy of the technique was tested under uni-axial tension and out of plane shear with both cyclic loading and modified monotonic loading conditions. This allowed the accuracy of the technique to be determined when applied to an industrial test specimen under different loading conditions.

Under uni-axial tension tests, pressure forces were applied to the ends of the specimen. For the cyclic loading analysis, a cyclic load range of $\pm 250 \mathrm{MPa}$ was applied and for the 
equivalent Modified Monotonic Loading analysis, a single monotonic pressure load of $+500 \mathrm{MPa}$ was applied. Under out of plane shear loading, pressure forces were applied to the specimen above and below the notch. For the cyclic loading analysis, a cyclic load range of $\pm 300 \mathrm{MPa}$ was applied. For the corresponding MML analysis, a monotonic pressure load of $+600 \mathrm{MPa}$ was applied. Diagrams showing the location of the applied forces for uni-axial tension and out of plane shear are shown in Fig 3 and Fig 4 respectively.

These analyses were merely comparative in order to visualise the differences between the cyclic loading and MML method.

Following successful validation, additional tensile tests and out of plane shear tests were performed in order to calculate the cyclic J-Integral using the proposed method.
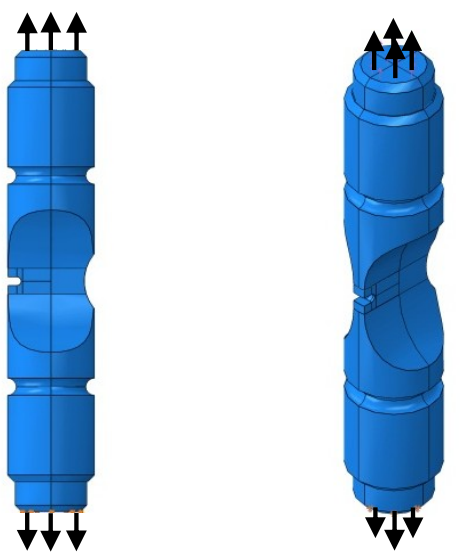

Fig 3: Uni-axial tension - location of loads
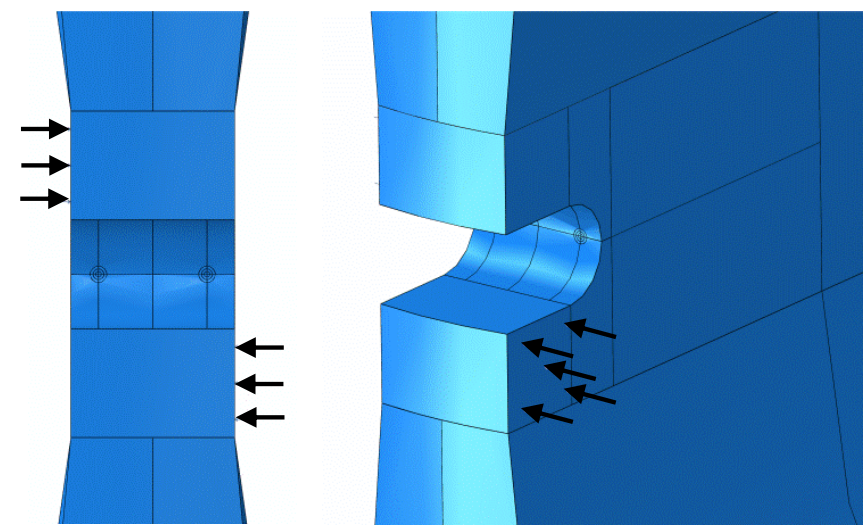

Fig 4: Out of plane shear - location of loads

\section{$5 \quad$ RESULTS AND DISCUSSION}

\subsection{Validation of MML Method}

The MML results were compared to the cyclic loading analysis to assess the suitability of the technique as a method of calculating the cyclic J-Integral. The stress range and strain range data from the cyclic loading analysis was compared to the stress and strain data of the MML method. Fig 5 to Fig 8 show stress and stress range and strain and strain range data from the MML and cyclic loading analyses for the uni-axial tension test. Fig 9 to Fig 12 show stress and stress range and strain and strain range data from the MML and cyclic loading analyses for the out of plane shear test

It can be seen that the differences between cyclic and MML analyses are minimal as the contour plots match very closely. As is assumed in (4) to (6), if the stress and strain data of the MML method matches the stress range and strain range data of the cyclic analysis, then the MML can be assumed to be a reasonable approximation of the cyclic J-Integral.

This technique means that a simple monotonic analysis is capable of determining the cyclic J-Integral. This offers a quick and computationally inexpensive method of determining the $\Delta \mathrm{J}$, which would otherwise be difficult and time consuming to calculate. From (2), it can offer an invaluable method of predicting the EPFM fatigue life of a component.

Some minor discrepancies exist between the contour plots of the MML and the cyclic loading analysis, however these differences are small and so the results are believed to be reasonable. It is felt that the speed and ease of implementation and calculation of this technique far outweighs any loss in accuracy.
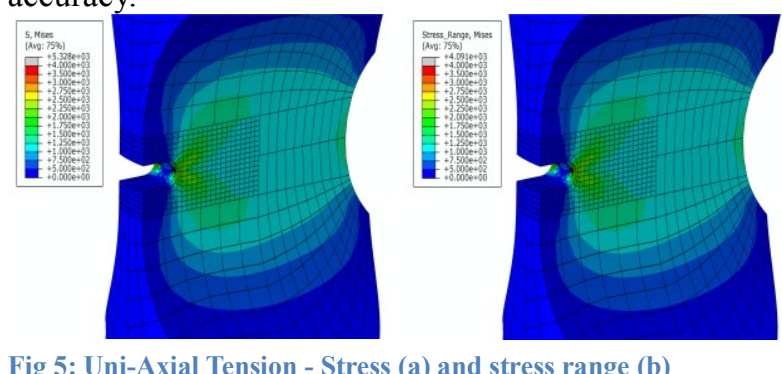

Fig 5: Uni-Axial Tension - Stress (a) and stress range (b)
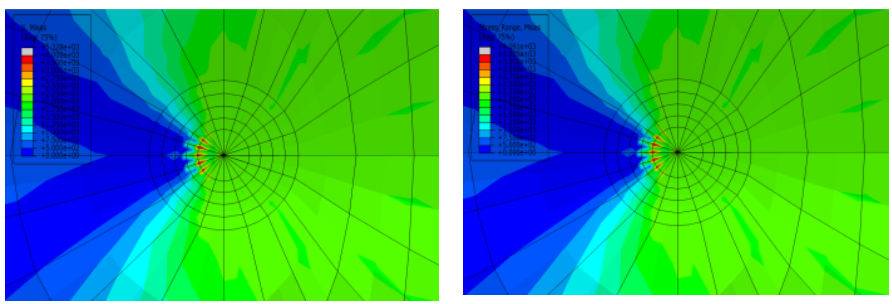

Fig 6: Uni-Axial Tension - Stress (a) and stress range (b) crack tip close up
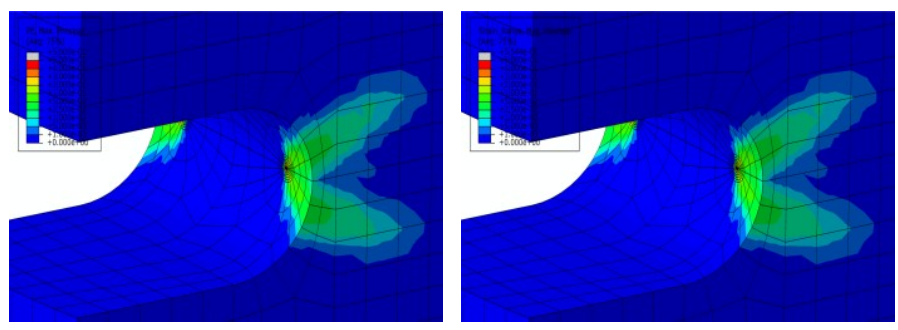

Fig 7: Uni-Axial Tension - Strain (a) and strain range (b) 

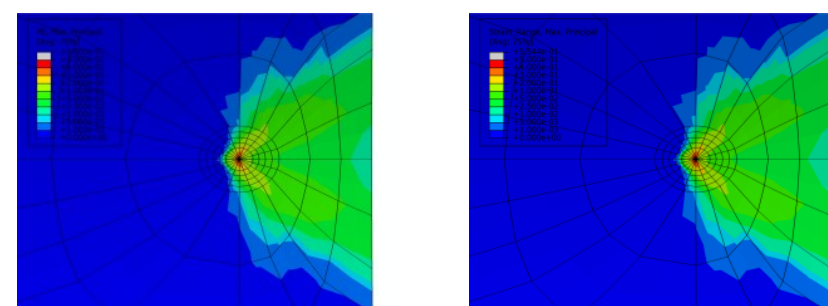

Fig 8: Uni-Axial Tension - Strain (a) and strain range (b) crack tip close up

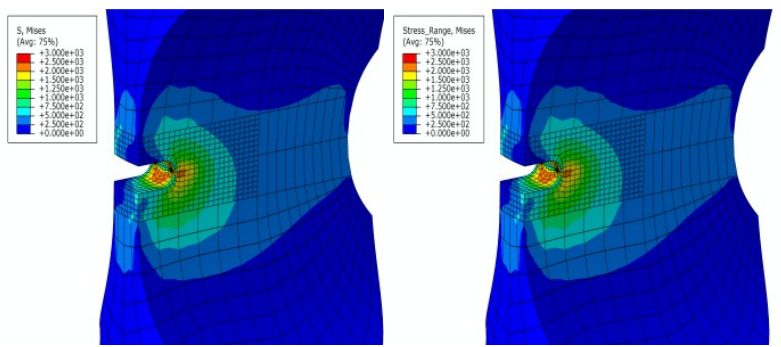

Fig 9: Out of plane shear - Stress (a) and stress range (b)
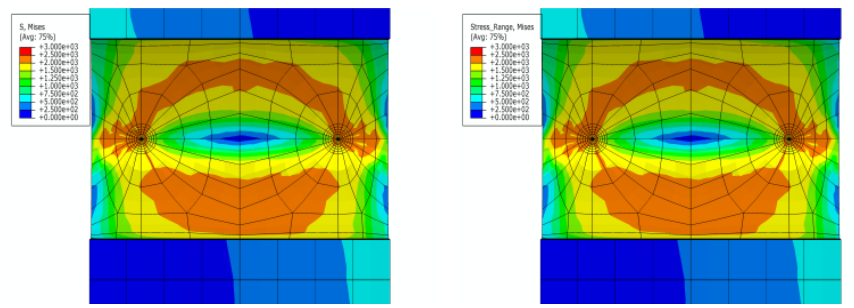

Fig 10: Out of plane shear - Stress (a) and stress range (b) crack tip close up
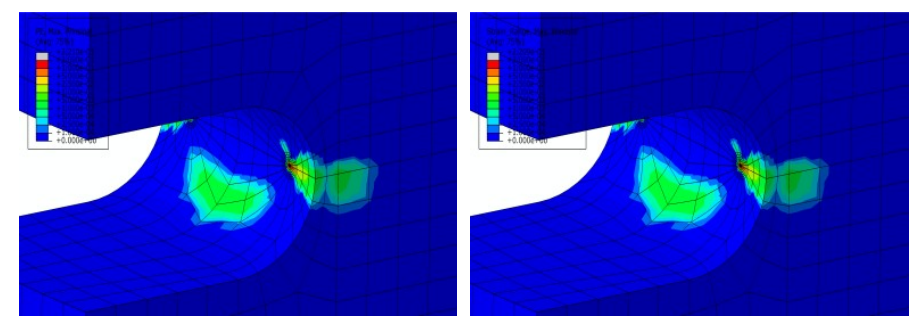

Fig 11: Out of plane shear - Strain (a) and strain range (b)
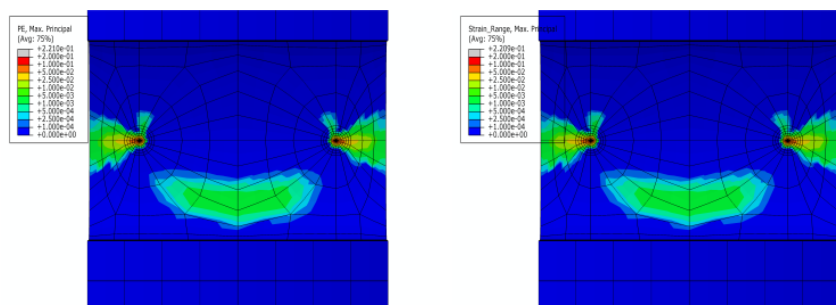

Fig 12: Out of plane shear - Strain (a) and strain range (b) crack tip close up

\subsection{Determination of cyclic J-Integral using MML}

An additional uni-axial tension test and out of plane shear test were performed on the specimen and the induced cyclic JIntegral recorded. The $\Delta \mathrm{J}$ at different locations along the crack was monitored as well as the variation of $\Delta \mathrm{J}$ with applied load.

The number of nodes that are defined along the crack front are assigned through the mesh refinement. For this investigation, the employed meshing rules implemented 25 nodes along the circumference of the crack front. Fig 13 is a schematic diagram of the crack front showing the node locations and numbers. Node 1 is located at the far left crack edge and Node 25 is located at the far right crack edge when facing the crack opening. These node numbers are referred to in the results in Table 1, Table 2, Fig 17 and Fig 18. For each node along the crack front, the $\Delta \mathrm{J}$ was calculated at 5 contours encircling the crack front. This is illustrated in Fig 14. The 5 contours at each node were averaged to give a single average value at each node along the crack front. This is believed to be accurate since the J-Integral is a path independent parameter[3], meaning that the value is the same, regardless of the path along which it is calculated. Within ABAQUS, numerical errors due to crack tip singularities mean that the values at each contour are not exactly equal, but any differences are very small and so average value is believed to be sufficiently accurate. These single values at each node were then tabulated and the average and maximum recorded, as well as far left end of crack, centre of crack and far right end of crack, shown in Table 1 for uni-axial tension, and Table 2 for out of plane shear. The J-Integrals provided in Table 1 and Table 2 and are obtained by the traditional elastic plastic fracture mechanics FEA using the MML concept. Therefore, these monotonic J-integrals are equivalent to cyclic J-integrals when the applied load range is equal to the magnitude of the load in the tables.

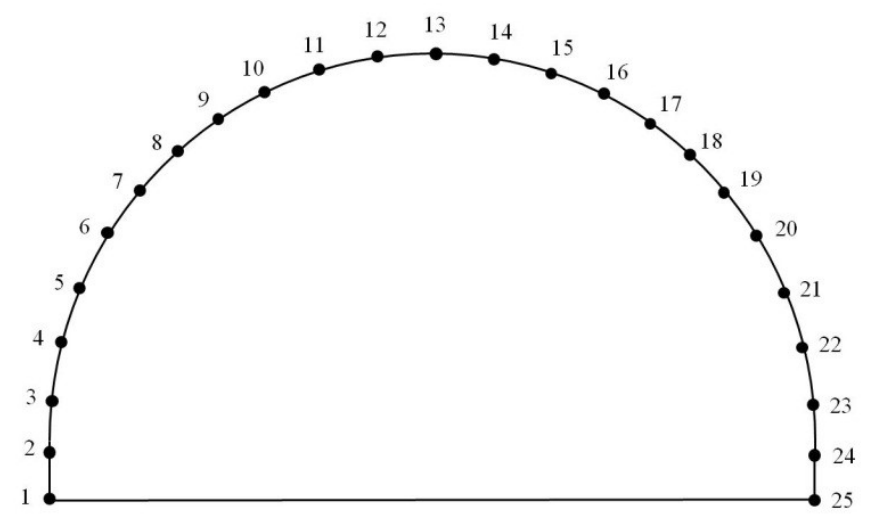

Fig 13: Crack Node Schematic

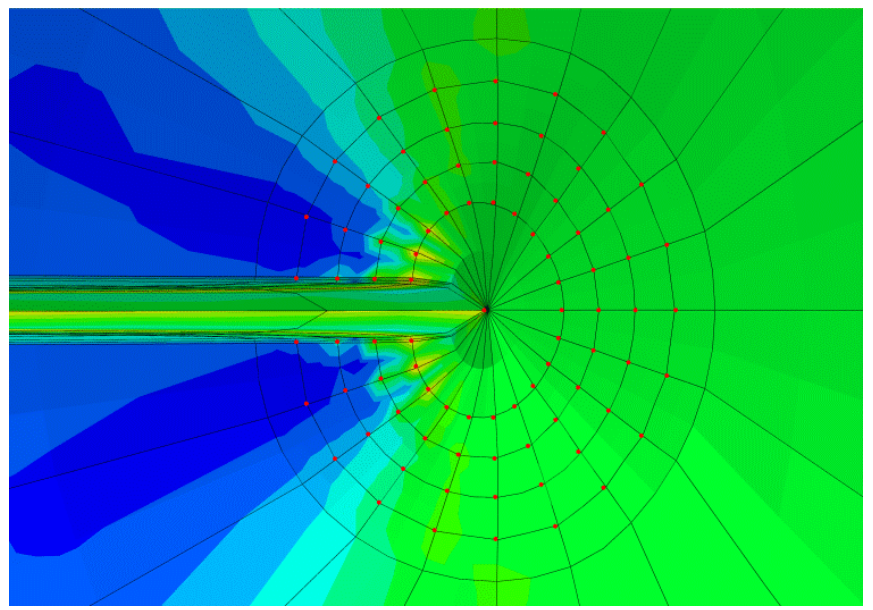

Fig 14: Contour Integral View 
Table 1: J-Integral Variation with Increasing Uni-Axial Load

\begin{tabular}{|c|c|c|c|c|c|}
\hline \multirow{2}{*}{$\begin{array}{c}\text { Load } \\
(\mathrm{MPa})\end{array}$} & Average & Max & $\begin{array}{c}\text { Jrack } \\
\text { Left } \\
\text { Node } 1)\end{array}$ & $\begin{array}{c}\text { Crack } \\
\text { Centre } \\
\text { (Node 13) }\end{array}$ & $\begin{array}{c}\text { Crack } \\
\text { Right } \\
\text { Node 25) }\end{array}$ \\
\hline 25 & $1.91 \mathrm{E}-03$ & $4.09 \mathrm{E}-03$ & $4.09 \mathrm{E}-03$ & $1.11 \mathrm{E}-03$ & $4.09 \mathrm{E}-03$ \\
\hline 50 & $7.63 \mathrm{E}-01$ & $1.60 \mathrm{E}+00$ & $1.60 \mathrm{E}+00$ & $4.42 \mathrm{E}-01$ & $1.60 \mathrm{E}+00$ \\
\hline 100 & $2.99 \mathrm{E}+00$ & $5.52 \mathrm{E}+00$ & $5.34 \mathrm{E}+00$ & $1.78 \mathrm{E}+00$ & $5.34 \mathrm{E}+00$ \\
\hline 150 & $6.67 \mathrm{E}+00$ & $1.19 \mathrm{E}+01$ & $9.87 \mathrm{E}+00$ & $4.06 \mathrm{E}+00$ & $9.87 \mathrm{E}+00$ \\
\hline 200 & $1.21 \mathrm{E}+01$ & $2.20 \mathrm{E}+01$ & $1.54 \mathrm{E}+01$ & $7.49 \mathrm{E}+00$ & $1.54 \mathrm{E}+01$ \\
\hline 250 & $1.97 \mathrm{E}+01$ & $3.50 \mathrm{E}+01$ & $2.22 \mathrm{E}+01$ & $1.25 \mathrm{E}+01$ & $2.22 \mathrm{E}+01$ \\
\hline 300 & $2.97 \mathrm{E}+01$ & $5.05 \mathrm{E}+01$ & $3.03 \mathrm{E}+01$ & $1.99 \mathrm{E}+01$ & $3.03 \mathrm{E}+01$ \\
\hline 350 & $4.22 \mathrm{E}+01$ & $6.85 \mathrm{E}+01$ & $4.02 \mathrm{E}+01$ & $3.05 \mathrm{E}+01$ & $4.01 \mathrm{E}+01$ \\
\hline 400 & $5.75 \mathrm{E}+01$ & $8.94 \mathrm{E}+01$ & $5.19 \mathrm{E}+01$ & $4.55 \mathrm{E}+01$ & $5.17 \mathrm{E}+01$ \\
\hline 450 & $7.69 \mathrm{E}+01$ & $1.16 \mathrm{E}+02$ & $6.68 \mathrm{E}+01$ & $6.68 \mathrm{E}+01$ & $6.65 \mathrm{E}+01$ \\
\hline 500 & $1.04 \mathrm{E}+02$ & $1.52 \mathrm{E}+02$ & $8.79 \mathrm{E}+01$ & $1.01 \mathrm{E}+02$ & $8.74 \mathrm{E}+01$ \\
\hline
\end{tabular}

Table 2: J-Integral Variation with Increasing Out of Plane Shear Load

\begin{tabular}{|c|c|c|c|c|c|}
\hline \multirow{2}{*}{$\begin{array}{c}\text { Load } \\
(\mathrm{MPa})\end{array}$} & Average & Max & $\begin{array}{c}\text { Jrack } \\
\text { Left } \\
\text { (Node 1) }\end{array}$ & $\begin{array}{c}\text { Crack } \\
\text { Centre } \\
\text { Node 13) }\end{array}$ & $\begin{array}{c}\text { Crack } \\
\text { Right } \\
\text { (Node 25) }\end{array}$ \\
\hline 25 & 0.000 & 0.000 & 0.000 & 0.000 & 0.000 \\
\hline 50 & 0.041 & 0.112 & 0.112 & 0.013 & 0.112 \\
\hline 100 & 0.166 & 0.449 & 0.449 & 0.053 & 0.446 \\
\hline 150 & 0.371 & 0.994 & 0.994 & 0.120 & 0.988 \\
\hline 200 & 0.661 & 1.759 & 1.759 & 0.215 & 1.748 \\
\hline 250 & 1.030 & 2.682 & 2.682 & 0.342 & 2.668 \\
\hline 300 & 1.480 & 3.750 & 3.750 & 0.502 & 3.732 \\
\hline 350 & 2.018 & 4.943 & 4.943 & 0.693 & 4.920 \\
\hline 400 & 2.649 & 6.260 & 6.260 & 0.927 & 6.233 \\
\hline 450 & 3.383 & 7.701 & 7.701 & 1.211 & 7.667 \\
\hline 500 & 4.232 & 9.274 & 9.274 & 1.562 & 9.234 \\
\hline
\end{tabular}

\section{Cyclic J-Integral Variation with Increasing Load}

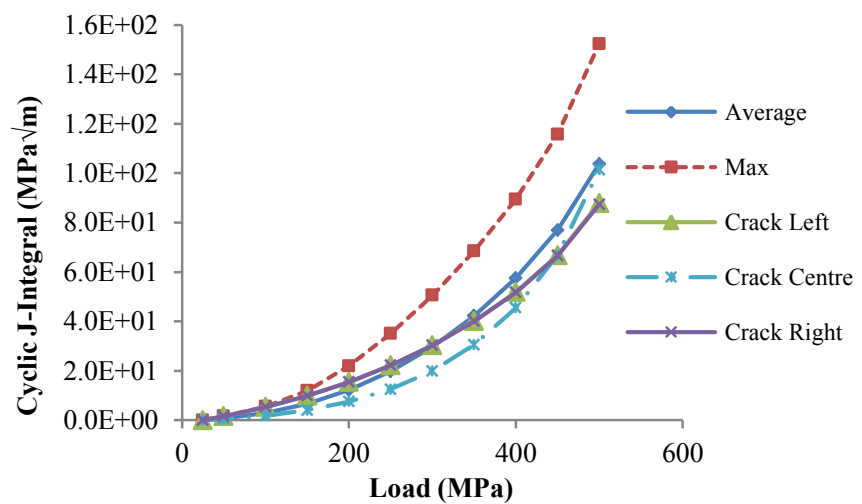

Fig 15: $\Delta J$-Integral Variation with Increasing Uni-Axial Load Graphical Representation

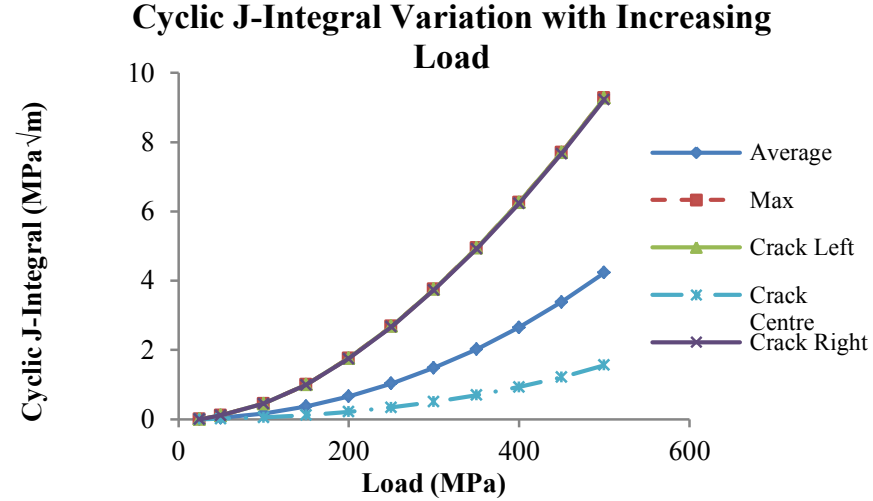

Fig 16: $\Delta \mathrm{J}$-Integral Variation with Increasing Out of Plane Shear Load Graphical Representation

Fig 15 and Fig 16 show a graphical representation of the cyclic J-Integral variation with increasing uni-axial and out of plane shear load at different locations along the crack front.

Under uni-axial loading (Fig 15), it can be seen that the average, crack left, crack centre and crack right agree closely due to the symmetry of the loading conditions, however, the maximum value is considerably higher. Upon closer investigation, it was found that the variation of the $\Delta \mathrm{J}$ along the crack front varies widely. Fig 17 shows this for applied uniaxial tension monotonic loads of $500 \mathrm{MPa}$ and $250 \mathrm{MPa}$, representing cyclic load ranges of $\pm 250 \mathrm{MPa}$ and $\pm 125 \mathrm{MPa}$ respectively. A maximum $\Delta \mathrm{J}$ value occurs slightly inside from the end of the crack, 3 nodes from each end and a minimum value occurs at 7 nodes from each end. The central region of the crack is relatively constant however the maximum and minimum values differ by a factor of 2 . The variation of rate of change of cyclic J-Integral with increasing load is not linear across the length of the crack. With increasing load, the maximum $\Delta \mathrm{J}$ values increase more rapidly and the minimum value increase more slowly relative to the values at the centre of the crack.

Under out of plane shear loading (Fig 16), it can be seen that the J-Integral relationship with increasing load is very different to that of uni-axial tension. Fig 18 shows the variation of $\Delta \mathrm{J}$ along the crack front under out of plane shear forces of $500 \mathrm{MPa}$ and $250 \mathrm{MPa}$, representing cyclic force ranges of $\pm 250 \mathrm{MPa}$ and $\pm 125 \mathrm{MPa}$ respectively. The maximum cyclic JIntegral coincides with the crack left and crack right positions with the crack centre being considerably less. This variation of $\Delta \mathrm{J}$ is due to the asymmetry of the loading conditions. This implies that the likeliest point of crack initiation under out of plane shear loading is at each side of the crack. The relationship is much more uniform than that of uni-axial tension with the maximum values occurring at the crack edge. The $\Delta \mathrm{J}$ steadily decreases along the crack front to a minimum at the centre with the maximum and minimum values differing by a factor of 5 . The rate of change of $\Delta \mathrm{J}$ along the crack front is much more uniform than that of uni-axial tension. 


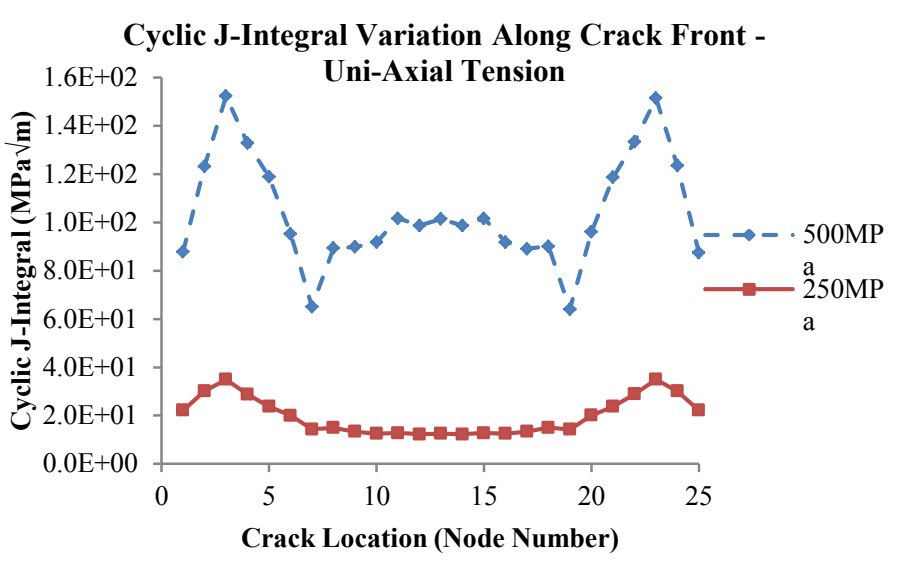

Fig 17: $\Delta \mathrm{J}$ Variation along Crack Front - Uni-Axial Tension

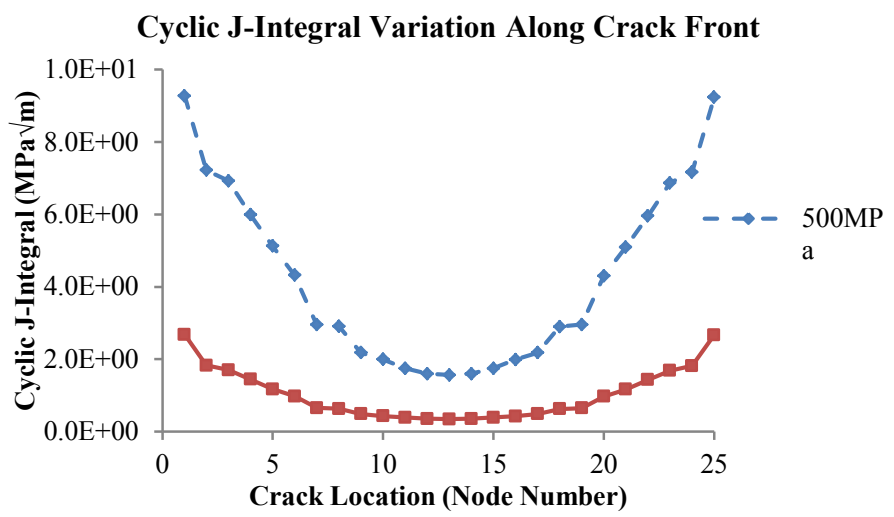

Fig 18: $\Delta \mathrm{J}$ Variation along Crack Front - out of plane shear

\subsection{Future Work}

This trend that has been noticed by the author will be the focus of future investigation to better understand $\Delta \mathrm{J}$ variation and thus the crack propagation direction. This will be compared to experimental results to better understand crack growth behaviour and assess the accuracy of these findings.

\section{CONCLUSIONS}

This study has proposed and validated the Modified Monotonic Loading method as a technique for the calculation of the cyclic J-Integral. A cyclic loading analysis was performed on an industrial test specimen under different loading conditions in order to establish a benchmark onto which the MML method can be validated. The MML method was then applied to the same test specimen under equivalent monotonic loading. The stress and stress range and strain and strain range data of the MML and cyclic loading analyses were compared, allowing the suitability of the technique to calculate the cyclic J-Integral to be ascertained. Once validated, additional analyses were performed on the test specimen which allowed the calculation of the cyclic J-Integral. Its variation with increasing load as well as along the crack front was recorded and the following observations have arisen:

1. Under uni-axial tension, the maximum $\Delta \mathrm{J}$ occurs slightly inside from the crack edge and the minimum being offset from the centre.

2. Under out of plane shear loading, the maximum $\Delta \mathrm{J}$ occurs at the crack edges, with the minimum being located at the crack centre.

These points of maximum $\Delta \mathrm{J}$ are likely points of crack initiation.

The application of this technique to an industrial test specimen has demonstrated its ability to determine the cyclic J-Integral of complex structures. The technique is inexpensive both in time and computational power and can be implemented with great ease offering a viable method of calculating the cyclic $\mathrm{J}$ Integral.

\section{ACKNOWLEDGMENTS}

The authors gratefully acknowledge the support of the Engineering and Physical Sciences Research Council (EPSRC) of the United Kingdom through the award of an EngD studentship, the Nuclear EngD Centre, the University of Strathclyde and Siemens Industrial Turbomachinery Ltd during the course of this work.

\section{REFERENCES}

[1] Irwin G (1957), Analysis of stresses and strains near the end of a crack traversing a plate, Journal of Applied Mechanics $24,361-364$.

[2] Griffith, A. A. (1921), The phenomena of rupture and flow in solids, Philosophical Transactions of the Royal Society of London, A 221: 163-198

[3] J. R. Rice, A Path Independent Integral and the Approximate Analysis of Strain Concentration by Notches and Cracks, Journal of Applied Mechanics, 35, 1968, pp. 379-386.

[4] R5, Assessment procedure for the high temperature response of structures, R5 Issue 3, June 2003, EDF Energy.

[5] R6 Revision 3, Assessment of the integrity of structures containing defects, British Energy Generation Ltd, Amendment 10, May 1999

[6] Dassault Systems Simulia Corp, 2012. Version 6.12-3 
[7] P Paris and F Erdogan (1963), A critical analysis of crack propagation laws, Journal of Basic Engineering, Transactions of the American Society of Mechanical Engineers, December 1963, pp.528-534.

[8] Sumpter JDG, Turner CE. Method for laboratory determination of JC (contour integral for fracture analysis). In: Cracks and fracture. ASTM STP 601. American Society for Testing and Materials; 1976.

[9] N. E. Dowling and J. A. Begley. Fatigue crack growth during gross plasticity and the J-integral. ASTM-STP WI. 1976; $82-103$.

[10] Chattopadahyay J. Improved J and COD estimation by GE/EPRI method in elastic to fully plastic transition zone. Eng. Fract Mech. 2006; 73. 1959

[11] A.G. Miller, and R.A. Ainsworth. Consistency of numerical results for power law hardening materials and the accuracy of the reference stress approximation. Eng Fract Mech. 1989; 32. 237

[12] Moës, N., Dolbow, J. and Belytschko, T. (1999), A finite element method for crack growth without remeshing. Int. J. Numer. Meth. Engng., 46: 131-150.

[13] Chen, W., Chen, H., Cyclic J-integral using the Linear Matching Method, International Journal of Pressure Vessels and Piping, Volumes 108-109, 2013, Pages 72-80,

[14] Leidermark, Daniel, et al. "A combined critical plane and critical distance approach for predicting fatigue crack initiation in notched single-crystal superalloy components." International Journal of Fatigue 33.10 (2011): 1351-1359. 